\title{
Cystoplasty Combined Autologous Peritoneum with Ileal Seromuscular segment: a new model study
}

\section{Jun Shen ( $\nabla$ surgery2020@163.com )}

Wuhan University Renmin Hospital

Junfeng Wu

Guizhou Medical University

Qinyue Zhao

Guizhou Medical University

Chunfeng Li

Beijng Huaxin Hospital First Hospital of Tsinghua University

\section{Yingmao Ruan}

Beijng Huaxin Hospital First Hospital of Tsinghua University

\section{Xi Chen}

Guizhou Medical University

\section{Yingmao Ruan}

Guizhou Medical University

\section{Research}

Keywords: Autologus peritoneum, lleal seromuscular segement, Cystoplasty, Model

Posted Date: May 22nd, 2020

DOI: https://doi.org/10.21203/rs.3.rs-27513/v1

License: (c) (i) This work is licensed under a Creative Commons Attribution 4.0 International License.

Read Full License 


\section{Abstract}

\section{Background}

After enterocystoplasty, some serious complications are presented, which are resulted from the chronic interaction between urine and intestinal epithelium. Composite cystoplasty is recommended as a method of surmounting these complications by replacing intestinal epithelium with autologous peritoneum.To develop a feasible surgical procedure for composite cystoplasty which can reduce serious complications postoperatively, keep sterility by excluding the possibility of contamination from the ileal contents, and provide a basis for the further clinical application of reconstruction bladder.

Methods

Six female Chinese minipigs (CMP) were randomly chosen, weighted $28-33 \mathrm{~kg}$. By intravenous anesthesia, bladder augmentation was carried out (implanting autologus peritoneum vascularized and, de-epithelialised ileal- seromuscular flaps. The composite cystoplasty used these flaps to mend and reconstruct bladders). Upon release of catheters and balloon at the fifth day, by monitoring the voiding behaviors of the pigs, routine pathology and immunohistochemistry were done by euthanizing the pigs at the 12th week.

Results

Six pigs experienced reconstruction, without complications. lleum was $10 \mathrm{~cm}$ long, and the area of substitute peritoneum was $7 \times 4 \mathrm{~cm}^{2}$. Voiding behavior was normal, and urine was clear in all animals after the catheter was taken out. Autopsy indicated that the fixed, bladder was in good condition, covered by continuous urothelium while the peritoneum disappeared, without regeneration of calculi, mucus, fibrosis, or ileal mucosa. Pathological examination demonstrated the success of the technique in developing the seromuscular flap without epithelial remnant. Bladders augmented with autologous peritoneum had fewer lymphocytes infiltration, indicating more effective urinary barrier function.

\section{Conclusion}

In this experimental porcine model, reconstruction bladder by autologous peritoneum and ileal seromuscular segment is an ideal approach since it can prevent regrowth of ileal epithelial cells and avoid the complications of conventional enterocystoplasty. The potential functions of the peritoneum are both protection and transitional support. This animal study provides a basis for further clinical application of the new enterocysplasty.

\section{Background}

While the treatment of the endstage diseases of bladders has adopted augmentation or reconstruction, using intestine to improve compliance and capacity is vulnerable to potentially high, and ordinarily serious complications as a result of continuous interaction between urine and absorptive, mucus- 
producing, and enteric epithelium ${ }^{[1-3]}$. Ureterocystoplasty, as alternatives to conventional enterocystoplasty, has proven to be of very limited use. As currently known, it seems the engineering of autologus tissue is the only reliable way to develop a urothelium-lined new bladder with sufficient function.

As described by some researchers in the concept of composite enterocystoplasty, autologous urothelium propagated in vitro combines with a segment of de-epithelialised bowel at surgical reconstruction ${ }^{[4]}$. By using the vascularised smooth-muscle tissue of patients with engineering of urothelium only in vitro, significant advantages are rendered over other strategies of tissue-engineering. These strategies are good, but they also have some deficiencies, such as the limitation of excision autologous urothelium, longer time to propagate in vitro, and reoperation. However, peritoneum has powerful function of restoration, which is advantageous in simple construction, abundant materials and easy excision. Some studies showed that peritoneum was applied in urinary tract or vagina reconstruction. Shaul et al ${ }^{[5]}$ used tubularized peritoneal free grafts as urethral substitutes in the rabbit, and at last, mesothelial cells of peritoneum were substituted by urothelium. Ait Sakel $A$ et $a^{\left[{ }^{[6]}\right.}$ successfully used peritoneal tube of Douglas' pouch for vaginoplasty in human body. Based on these facts, we replaced the ileal mucosa and submucosa with autologus peritoneal grafts, and used the new grafts for cystoplasty. We here presented the primitive results of the new method.

\section{Materials And Methods}

Six female Chinese minipigs (CMP) were aged 6-8 months and weighed $28-33 \mathrm{~kg}$. All the procedures were in compliance with standard protocols and statutory regulations. Preliminary operations were performed using fresh ileum, peritoneum and bladder of pigs from an abattoir. They were fasted from food and water for $24 \mathrm{~h}$ and $4 \mathrm{~h}$, respectively. They were premedicated with xylazine hydrochloride $2.0 \mathrm{mg} / \mathrm{kg} \mathrm{IM}$ and atropine sulfate injection $0.02 \mathrm{mg} / \mathrm{kg}$ following IM route. Anesthesia was done with $1 \mathrm{mg} / \mathrm{kg}$ intravenous propofone and $0.5 \mathrm{mg} / \mathrm{kg}$ atracurium besylate. After two minutes, the animals were intubated successfully, maintaining and anesthesia with isoflurane in $100 \%$ oxygen by use of a rebreathing circuit. Ventilation (Drag, Germany) was adopted to keep the level of endtidal carbon dioxide levels at $36-45 \mathrm{mmHg}$, the level of $\mathrm{SPO}_{2}$ at $98 \%-100 \%$, and the heart rate at $80-120 \mathrm{bpm}$. During the surgical process, anesthesia was assisted with propofone $5.0-10.0 \mathrm{mg} / \mathrm{kg} / \mathrm{h}$ and atracurium besylate $0.2-0.3 \mathrm{mg} / \mathrm{kg} / \mathrm{h}$ via IV pump, and sufentanil citrate (sufentanil) $5.0-10.0 \mathrm{mcg} / \mathrm{kg}$ was maintained every 40minutes for deep analgesia. Atracurium besylate was stopped 10-20 minutes before the end of surgery. In recovery, every animal's breath status was observed when the tube was withdrawn.

Upon cleaning of the surgical field with chlorohexidine and betadine, the bladder was inserted with a F14 Foley catheter. The abdomen was opened through an infraumblical incision. The peritoneum was identified and the fats around were removed dully. The enterocoelia was opened from one side of the peritoneum which was saved for the subsequent use. 
From approximately $15 \mathrm{~cm}$ distance to ileocecus backward, a flap of ileum about $10 \mathrm{~cm}$ long was prepared with vascular arcades in the mesentery. By placing stay sutures on the antimesenteric seromuscular border of the ileum beyond the dissection limits, mesenteric fenestrations were rendered with at least 2 branching vessels supplying the intestine from the vascular arcade. Inside the sutures, the seromuscular layer was incised by using a finger, and then, by using micro pliers and smooth forceps, we separated the seromuscular layer from the muscular to the submucosa with a dissection in between (Fig. 1-2). Dissection margins connected with mesenteric fenestrations at the mesenteric border to separate the seromuscular layer from the ileum remained by electrocoagulation. Stretched onto a board muscular layer up, the seromuscular layer of the pedicle flap was fixed with pushpins. Extramucosal ileal dissection was adopted to develop a vascularised and, de-epithelialised, seromuscular flap at the mean size of $25 \mathrm{~cm}^{2}$ (range, $20-28 \mathrm{~cm}^{2}$ ). The same area of peritoneum was cut, smoothed surface up, and covered onto the muscular layer (Fig. 3). Both of the edges were sutured with $6 / 0$ Vicryl. With the bladder opened wide and augmented with a composite intestine flap, its gentle distension was kept with a silicone balloon of the catheter, gently apposing the peritoneum to the intestine (Fig. 4). Urine passed urethra under the support of ureteric stents (Vygon 4Fig). After resecting the mucosa and submucosa with devascularization, the edges from the intestine remained were closed by using $3 / 0$ Vicryl. The mesenteric fenestration was also closed. With the abdomen closed, catheters and ureteric stents were secured externally with adhesive bandage. Postoperatively, they animals were fasted from food for $48 \mathrm{~h}$ but not from water. They had been injected with Benzylpenicillin $\mathrm{G}(0.48 \mathrm{~g}, \mathrm{q} 12 \mathrm{~h})$ for three days. To keep the ureteric stents clear, we used gentamicin dilute solution (1:500) to wash them slowly and extract (20 ml, q12h). To prevent retrograde infection, iodophor and its dilute solution (1:3) were used to gently flush external urethral orifice and catheter respectively. Catheters and ureteric stents were removed after 5 days. After sacrifice at week 12, bladders were collected and processed for routine pathology and immunohistology.

We trimmed bladder segments in $10 \%$ formalin for $24 \mathrm{~h}$, with tissues dehydrated through ethanol and embedded in paraffin wax. Four micrometre de-waxed sections were either stained with haematoxylin and eosin to shape the structure of augmented neobladders or labelled with antibodies. CK7 was used to shape the phenotype of the tissue. UPK3a was used as marker of urothelium. Calretini and HBME-1 were used to identify the peritoneum.

According to the principle of electron microscope, we remove bladder specimen and send it to Electron Microscope Room for scanning electron microscopy (SEM) and transmission electron microscopy (TEM) respectively.

\section{Results}

The postoperative period was smooth in six pigs, and urine drainage was fluent and clear. Normal voiding was reestablished after catheter removal. As per study design, the pigs were sacrificed at week 12 following the initial operation. All the animals witnessed intact urinary bladders (Fig. 5), and the vascular pedicles of the ileal grafts were normal, without evidence of perforation, leakage, inflammation, ulcers, 
mucus, or any other focal lesion. It seems that some augments witnessed contracted size, but without morphologic evidence of fibrosis. Shrinkage of visceral tissue might occur immediately after evisceration in air. Anastomosis of both recovered and the luminal surface upon resection showed smooth and intact (Fig. 6).

Histologically, a clear demarcation appeared between the smooth muscle of bladder and intestine at the anastomosis. A continuous urinary transitional epithelium appeared in all the tissues, both in the origina bladder and the augmented sections (Fig. 7). In morphology, there appeared no regeneration of of ileal epithelial cells and goblet cells in any of the bladders augmented. While there was a little density of lymphocytic infiltration in the lamina propria in the bladders treated, inflammation was minimal.

There was some CK7 and UPK3a was detected in urothelium, rather than in peritoneal tissues. These same isotypes appeared in the superficial edge of native epithelium in all augmented flaps (Fig. 8, 9). Especially, UPK3a appeared in all augmented epithelia, indicating a transitional phenotype and absence of peritoneal tissue. Moreover, as the special antibody, Calretini and HBME-1 should be detected in peritoneal tissues, but they were negative in all augmented segments covered with peritoneum during operation (Fig. 10).

Ultrastructural observation under electron microscope: Fig. 11 shows SEM results of normal bladder mucosa: Bladder mucosal epithelial cells are long fusiform, rounded edges, small gaps between cells, and dense arrangement. Neobladder mucosal epithelium structure postoperatively 12 weeks (Fig. 12): The overall structure is complete; mucosa cells are oval, which edges are blunt; the connecting gaps between individual cells become smaller; and the arrangement is relatively loose. To be observed by transmission electron microscope, as Fig. 13 shown, the normal ileum smooth muscle cells are spindle shaped, and closely connected with each other, which boundaries are clear, and arranged neatly. The nucleus in the center of the cell is similar to the shuttle shape which is normal; the point-like dense spot is clearly visible; the base membrane is complete; and only a few fiber filaments are seen in the interstite. The ultrastructure of the neobladder muscle fibroblasts 12 weeks postoperatively is shown in Fig. 14: smooth muscle cells are spindle shaped, arranged neatly; there is no obvious hypertrophy; and the base membrane and dense spots are clearly visible. The cytoplasmic components do not fall off, which realm is clear; the mitochondrial morphology is not abnormal, such as neither aggregation nor swelling; and there is no obvious proliferation of mesenchymal fibers.

\section{Discussion}

Although the skill of enterocystoplasty was better and better, the nature of intestine for reconstructing bladder was unchanged. Therefore, urologists and patients had always been surrounded by the postoperative complications associated with secretion and absorption of intestine epithelium. Although some researchers found methods, such as striking off mucosa by scalpel, corroding it by absolute ethyl alcohol, etc., to destroy intestine mucosa so as to decrease secretion and absorption of intestine mucosa, the effects were unsatisfactory. Comparatively speaking, we striped ileal seromuscular segment entirely, 
and used autologous peritoneum covering upon it, which can avoid the complications and protect the muscle to be stimulated by urine. In this study, we displayed a successful composite cystoplasty of bladder reconstruction in pigs. Soon after surgery appeared normal voiding, and examination of the fixed bladders 12 weeks later witnessed viable, vascularised, cystoplasty segments without fibrosis or contraction. Augmented segments lined by confluent urothelium witnessed minimal inflammatory changes, showing effective barrier function from the peritoneum to the urothelium. Especially in the experiment, it was demonstrated that the extraluminal dissection of the ileum facilitated the formation of robust seromuscular segment without ileal goblet cells and epithelial regeneration in pigs. Our technique features direct exfoliation of ileal seromuscular segment by extraluminal dissection other than using Foley catheter and the balloon to facilitate the dissection any more. Maybe the balloon distended well to sigmoid colon ${ }^{[4,7,8]}$, but it was not suitable for ileum. The reasons are as follows: firstly, sigmoid colon is closer to anus than ileum; secondly, the lumen of sigmoid colon is bigger than that of ileum; thirdly, the ileal well is thinner than that of sigmoid colon. Hafez et al ${ }^{[9]}$ also used this distending technique, but under the help of hydrodistension. They had the colonic segment filled with water, hence striping the seromuscular flap. Contamination of the operative field may be caused potentially, if the mucosa is perforated, which can be prevented by our method. Therefore, as one of our aims, we tried to avoid such problems as previously occurred in dissecting the thin porcine ileum ${ }^{[10]}$. By using a modified technique ${ }^{[11]}$, we could create a properly vascularised seromuscular segment of viability by combining with autologous peritoneum. At sacrifice there appeared no contraction in the augmented segment, or fibrosis, but healthy smooth muscle. Importantly, a clean plane of separation appeared under the level of submucosa, hence preventing regeneration of ileal mucosal-cell and goblet cell from the basal layers. Compared to pigs, the muscle wall of human ileum is also thick and a similar feasibility of demucosalisation has been established $^{[11]}$. Nevertheless, the adoption extramucosal dissection may reduce the risk of sepsis by preventing peritoneal soiling with intestinal content. Fraser et al ${ }^{[4,12]}$ found that intestinal mucosal regeneration was a fearful problem, hence suggesting excision of muscularis mucosae with the inner side of the submucosa to prevent this troublesome complication. However, they peeled the mucosa from within the lumen after cutting out the intestinal section. Similar outcomes of enteric epithelium regeneration appeared in seromuscular colocystoplasty by others ${ }^{[13]}$. Afterwards, Subramaniam et al ${ }^{[7]}$ removed the colonic mucosa and muscularis mucosae at the level of submucosa from extralumen, and found no evidence of enteric regrowth. Compared to colon, ileal submucosa was thin and, and its muscularis mucosae was incomplete somewhere. Here our study showed that the naked surface of muscle after the mucosa and submucosa removal was beneficial for the survival of the peritoneum transplanted. As our study showed, it bled scarcely on operation of stripping. To prevent this problem, we regard extraluminal dissection a much simpler and safer procedure.

Lima et al ${ }^{[8]}$ also adopted an intravesical balloon, significantly reducing fibrosis in the canine portion, and they commented in other studies that the presence of an epithelial lining seemed to prevent fibrosis and contraction. In our study, we adopted an intravesical balloon to provide for apposition of the peritoneum, supporting the peritoneum to the seromuscular flap ${ }^{[14]}$. 
On one hand, we indicated that UPK3a and CK7 appeared in the epithelium of all our augmented segments, displaying a transitional phenotype with urothelium development, without ileal epithelial regeneration; On the other hand, Calretini and HBME-1 were negatively expressed, illustrating a phenotype

with no peritoneum survival. Although De Badiola et al ${ }^{[15]}$ and Lima et al ${ }^{[16]}$ reported that the seromuscular cystoplasty technique was feasible in humans, which avoided some postoperative complications, and the ultimate results would be unsatisfactory if the surface of muscle interacted with urine permanently, without anything to be protected. In humans, UPK3a and CK7 provide an objective marker of late/terminal urothelial differentiation, the expression of which parallels the development of a functional barrier. In light of electromicroscopy, we can observe that upon the smooth muscle of neobladder are completely covered by urothelial cells, which the smooth muscle cells are protected well. During the 12-week experiment, the peritoneum covering upon seromuscular segment of ileum was substituted by urothelium gradually. This highlighted that while the peritoneum was absorbed little by little, it was a barrier for urine stimulating muscle and an underprop for crawling urothelium. Although swine is valuable as model for the development and test of surgical procedures, it cannot directly substitute studies on human body.

\section{Conclusions}

A successful application of composite enterocystoplasty to pigs has been depicted, including a reconstructing ileal smooth-muscle segment covered by autologous peritoneum which prevented the muscle to be eroded by urine and put itself as an upholder for urothelium. None of well-grounded complications of the conventional enterocystoplasty was discovered in the reconstructed neobladders.

This animal study provides the basic ground for further clinical application of enterocysplasty. We suggest the approach may be applied in human beings once it is approved by the ethics committee.

\section{Abbreviations}

SEM

Scanning electron microscopy

TEM

Transmission electron microscopy

CMP

Chinese minipigs

\section{Declarations}

\section{Availability of data and materials}

Materials are available upon request. 
I would like to thank the first affiliated hospital of guizhou medical university, the first affiliated hospital of tsinghua university and the department of urology of qiandongnan people's hospital affiliated to guizhou medical university for their support

\section{Funding}

The Joint Foundation of Guiyang Science and Technology Bureau and Guizhou Medical University [No. GY2017-16]; 2. The Doctoral Scientific Foundation of Guizhou Medical University (No. YJ2017-18); 3. Science and Technology Program of Guizhou Province『No. [2018]5779凶6囚.

\section{Financial disclosures}

I certify that all conflicts of interest, including specific financial interests and relationships and affiliations relevant to the subject matter or materials discussed in the manuscript (eg, employment/ affiliation, grants or funding, consultancies, honoraria, stock ownership or options, expert testimony, royalties, or patents filed, received, or pending), are the following: None.

\section{Author information}

Junfeng Wu and Qinyue Zhao contributed equally to this work.

\section{Affiliations}

Junfeng Wu[Department of Urology,, Qiandongnan people's hospital affiliated to guizhou medical university $\llbracket$ Kaili『People's Republic of China $\$ 556000$

Qinyue Zhao $\mathbb{D}$ Department of Anesthesiology, Guiqian International General Hospital, Guiyang, People's Republic of China, 550018

Chunfeng Li, Yingmao Ruan $\mathbb{D}$ epartment of Pathology, the First Hospital of Tsinghua University, Beijing, People's Republic of China, 100000

Xi Chen, Peng Chen, Jun ShenロDepartment of Urology, the Affiliated Hospital of Guizhou Medical University, Guiyang, People's Republic of China,550004

\section{Contributions}

Conception and design: Jun Shen; Development of methodology: Junfeng Wu,Qinyue Zhao; Acquisition of data: Junfeng Wu,Qinyue Zhao; Analysis and interpretation of data:Junfeng Wu,Qinyue Zhao, Chunfeng Li, Yingmao Ruan; Writing, review, and/or revision of the manuscript: Junfeng Wu,Qinyue Zhao,Jun Shen; Administrative, technical, or material support:Xi Chen, Peng Chen, Jun Shen; Study supervision:Xi Chen, Peng Chen, Jun Shen. The authors read and approved the final manuscript.

\section{Corresponding author}


Correspondence to Jun Shen.

\section{Ethics declarations}

\section{Ethics approval and consent to participate}

Animal experiments were approved by the medical research animal ethics committee of guizhou medical university.

\section{Consent for publication}

Not applicable.

\section{Competing interests}

The authors have declared no conflicts of interest.

\section{References}

1. Nicita G, Martini A, Filocamo MT, et al Use of sigmoid colon in orthotopic neobladder reconstruction: Long-term results. Int J Urol. 2016. 23(12): 984-990.

2. Prcic A, Begic E. Complications After Ileal Urinary Derivations. Med Arch. 2017. 71(5): 320-324.

3. Yadav SS, Gangkak G, Mathur R, Yadav RG, Tomar V. Long-term Functional, Urodynamic, and Metabolic Outcome of a Modified Orthotopic Neobladder Created With a Short Ileal Segment: Our 5year Experience. Urology. 2016. 94: 167 - 72.

4. Fraser M, Thomas DF, Pitt E, Harnden P, Trejdosiewicz $L K$, Southgate J. A surgical model of composite cystoplasty with cultured urothelial cells: a controlled study of gross outcome and urothelial phenotype. BJU Int. 2004. 93(4): 609 - 16.

5. Shaul DB, Xie HW, Diaz JF, Mahnovski V, Hardy BE. Use of tubularized peritoneal free grafts as urethral substitutes in the rabbit. J Pediatr Surg. 1996. 31(2): 225-8.

6. Ait Sakel A, Asseban M, Kallat A, et al [Vaginoplasty from peritoneal tube of Douglas' poutch for androgen insensitivity syndrome (two case reports)]. J Gynecol Obstet Biol Reprod (Paris). 2014. 43(6): 470-3.

7. Subramaniam R, Turner AM, Abbas SK, Thomas DF, Southgate J. Seromuscular grafts for bladder reconstruction: extra-luminal demucosalisation of the bowel. Urology. 2012. 80(5): 1147-50.

8. Lima SV, Araújo LA, Vilar FO, Kummer CL, Lima EC. Nonsecretory sigmoid cystoplasty: experimental and clinical results. J Urol. 1995. 153(5): 1651-4.

9. Hidas G, Lee HJ, Bahoric A, et al Aerosol transfer of bladder urothelial and smooth muscle cells onto demucosalized colonic segments for bladder augmentation: in vivo, long term, and functional pilot study. J Pediatr Urol. 2015. 11(5): 260.e1-6. 
10. von Trotha KT, Butz N, Grommes J, et al Vascular anatomy of the small intestine-a comparative anatomic study on humans and pigs. Int J Colorectal Dis. 2015. 30(5): 683 - 90.

11. Hafez AT, Bagli DJ, Bahoric A, et al Aerosol transfer of bladder urothelial and smooth muscle cells onto demucosalized colonic segments: a pilot study. J Urol. 2003. 169(6): 2316-9; discussion 2320.

12. Westfal ML, Goldstein AM. Pediatric enteric neuropathies: diagnosis and current management. Curr Opin Pediatr. 2017. 29(3): 347-353.

13. Dewan PA, Close CE, Byard RW, Ashwood PJ, Mitchell ME. Enteric mucosal regrowth after bladder augmentation using demucosalized gut segments. J Urol. 1997. 158(3 Pt 2): 1141-6.

14. Turner A, Subramanian R, Thomas DF, et al Transplantation of autologous differentiated urothelium in an experimental model of composite cystoplasty. Eur Urol. 2011. 59(3): 447 - 54.

15. De Badiola F, Ruiz E, Puigdevall J, et al Sigmoid cystoplasty with argon beam without mucosa. J Urol. 2001. 165(6 Pt 2): 2253-5.

16. Lima SV, Araujo LA, Vilar Fde O, Lima RS, Lima RF. Nonsecretory intestinocystoplasty: a 15-year prospective study of 183 patients. J Urol. 2008. 179(3): 1113-6; discussion 1116-7.

\section{Figures}




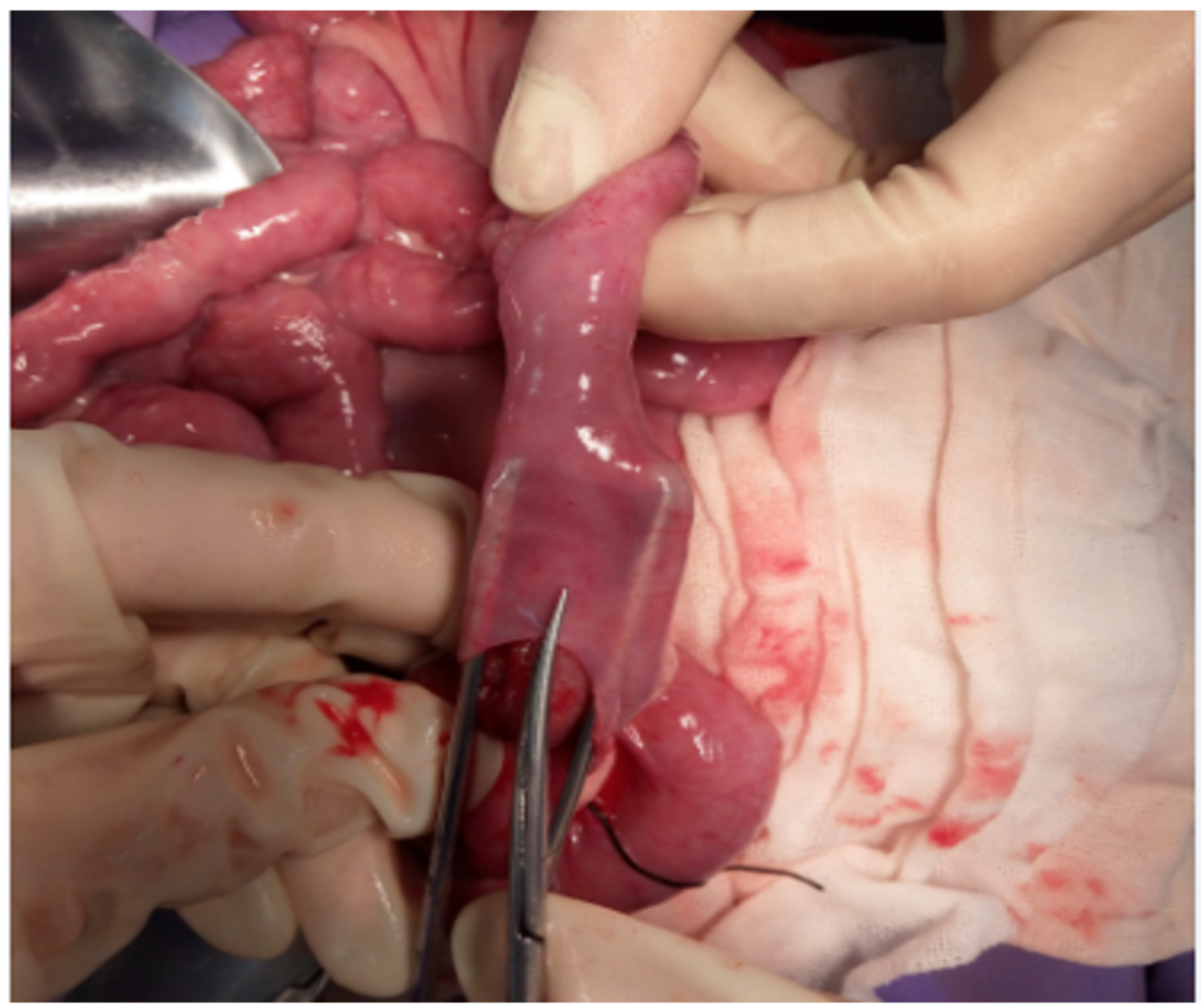

Figure 1

Extramucosal ileal dissection, by using smooth forceps, the seromuscular layer was separated dully. 


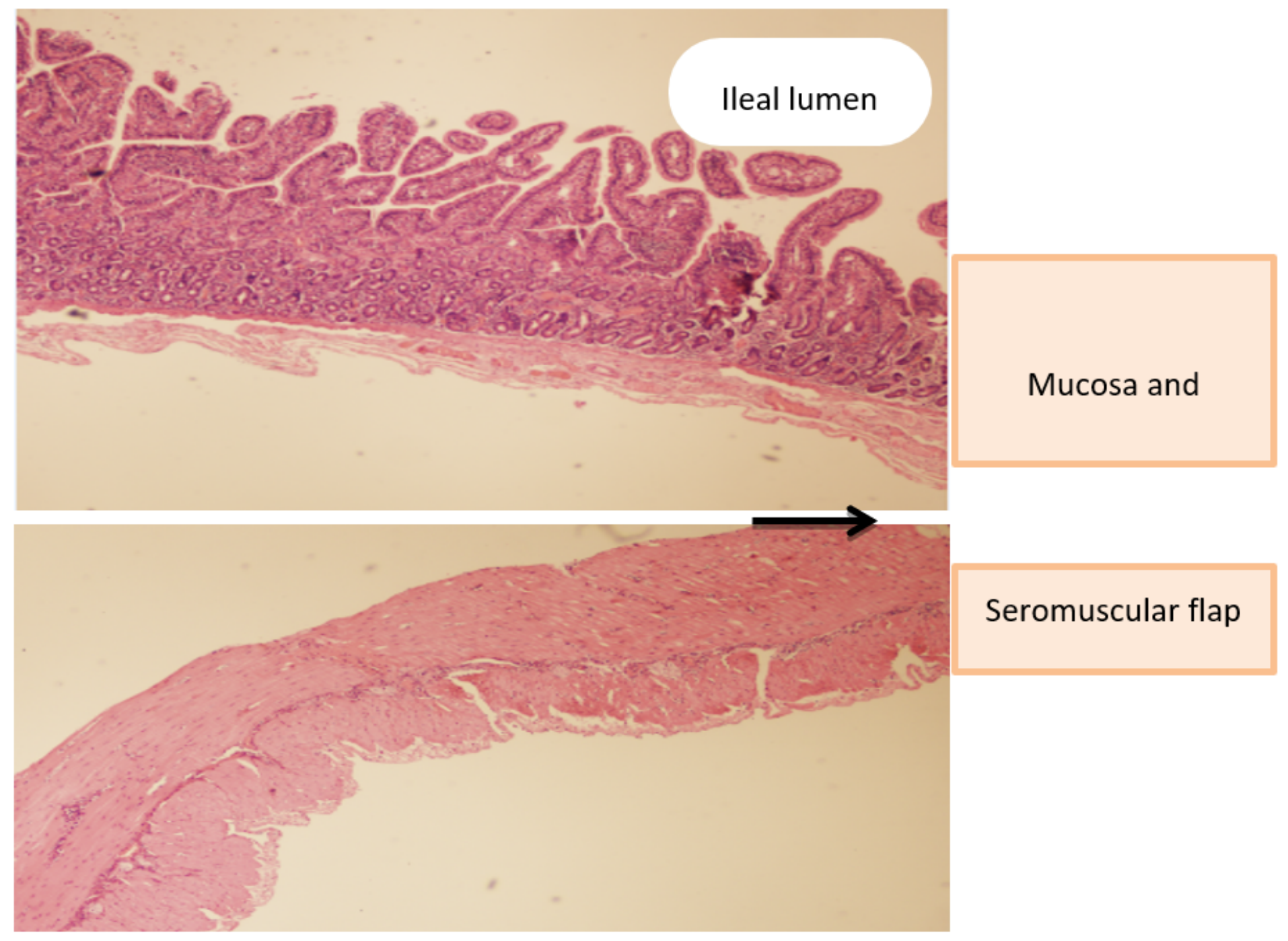

Figure 2

Plane of dissection on ileal well (arrow), (Hematoxylin-eosin staining, Low-power field) 


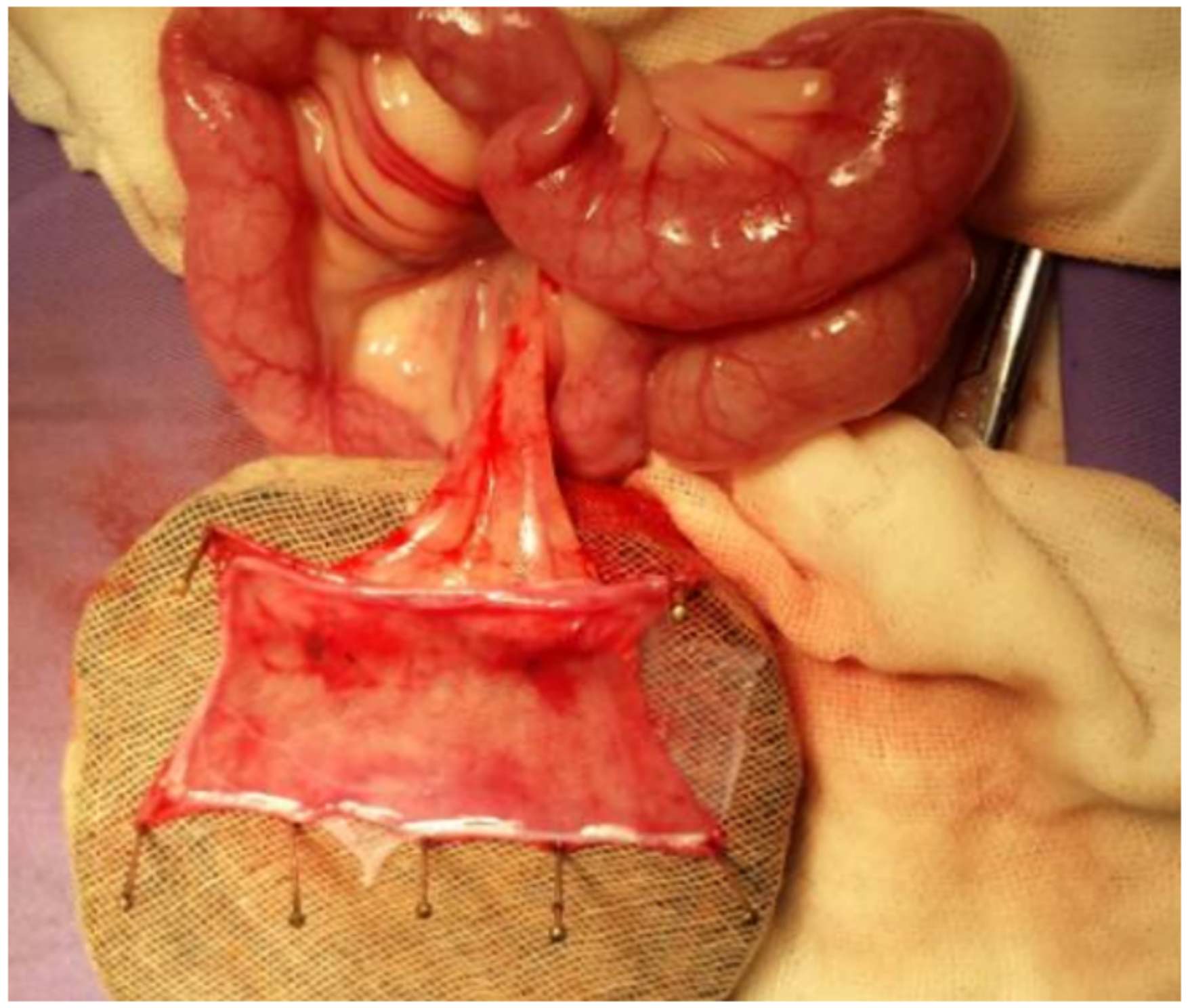

\section{Figure 3}

The peritoneum was covered onto the muscular layer. 


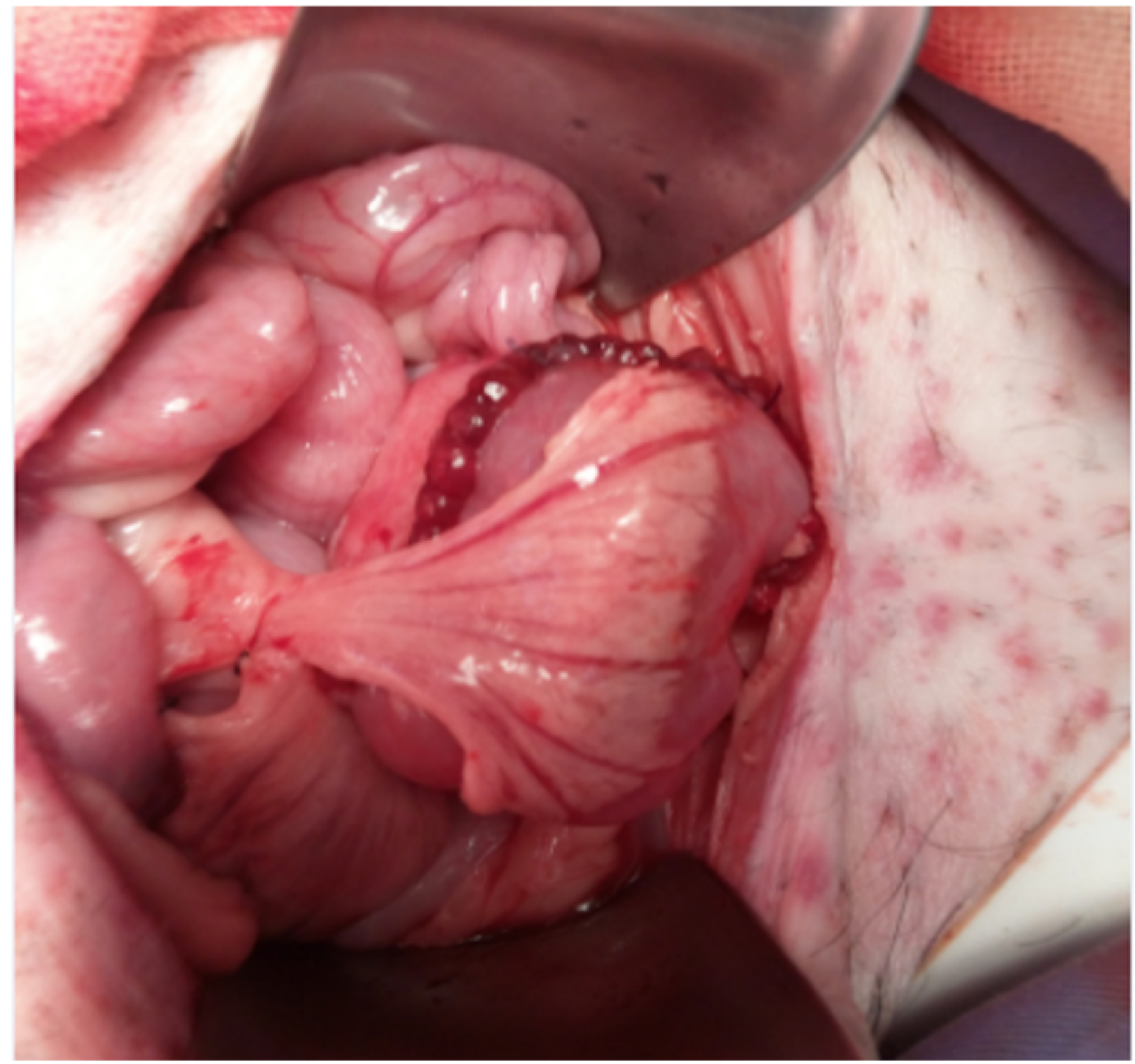

\section{Figure 4}

The augmented bladder with good vascularization, was maintained with a silicone balloon of the catheter. 


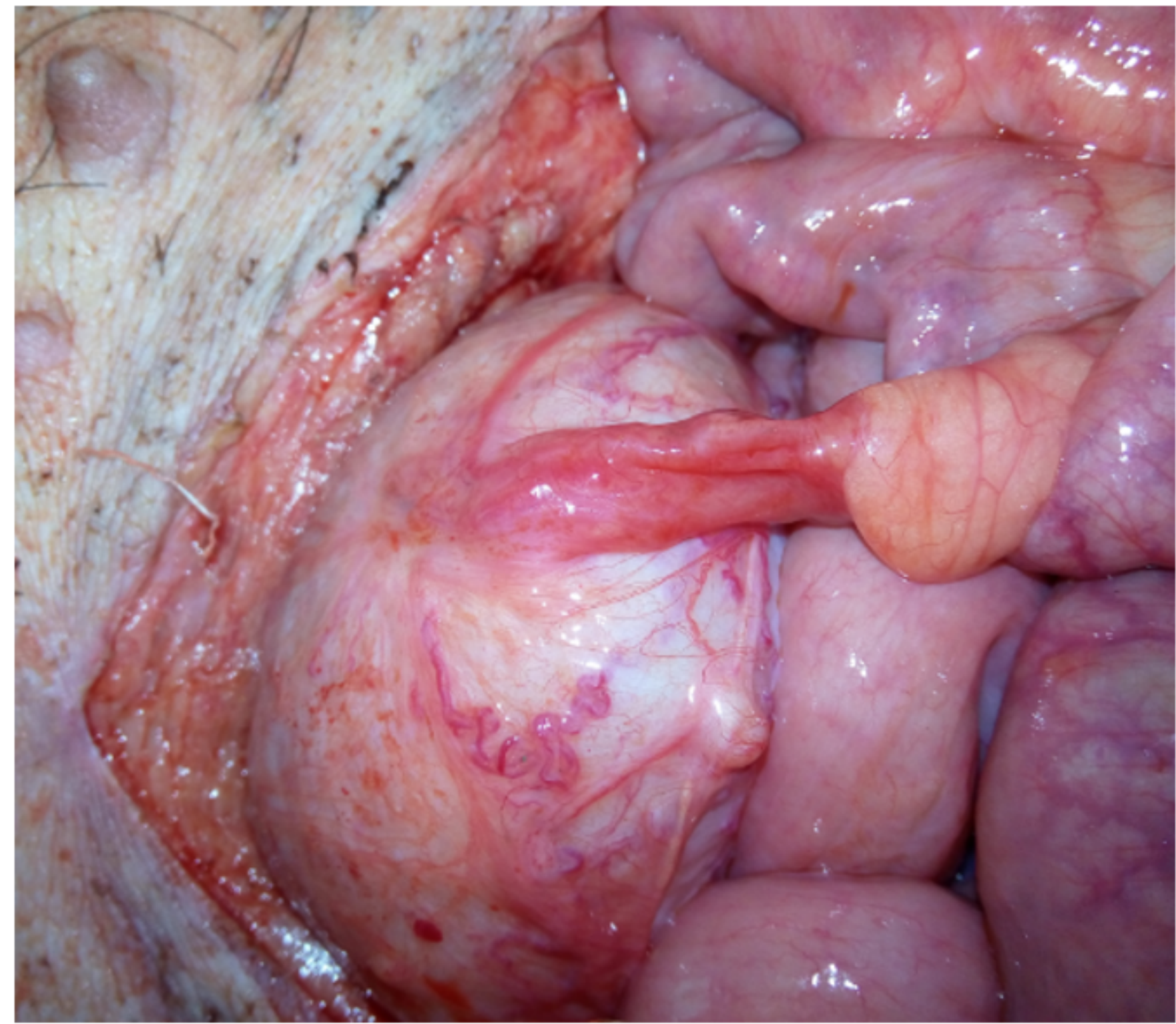

Figure 5

Postoperatively 12 weeks, an intact bladder was showed, and the vascular pedicle of the ileal grafts was normal. 


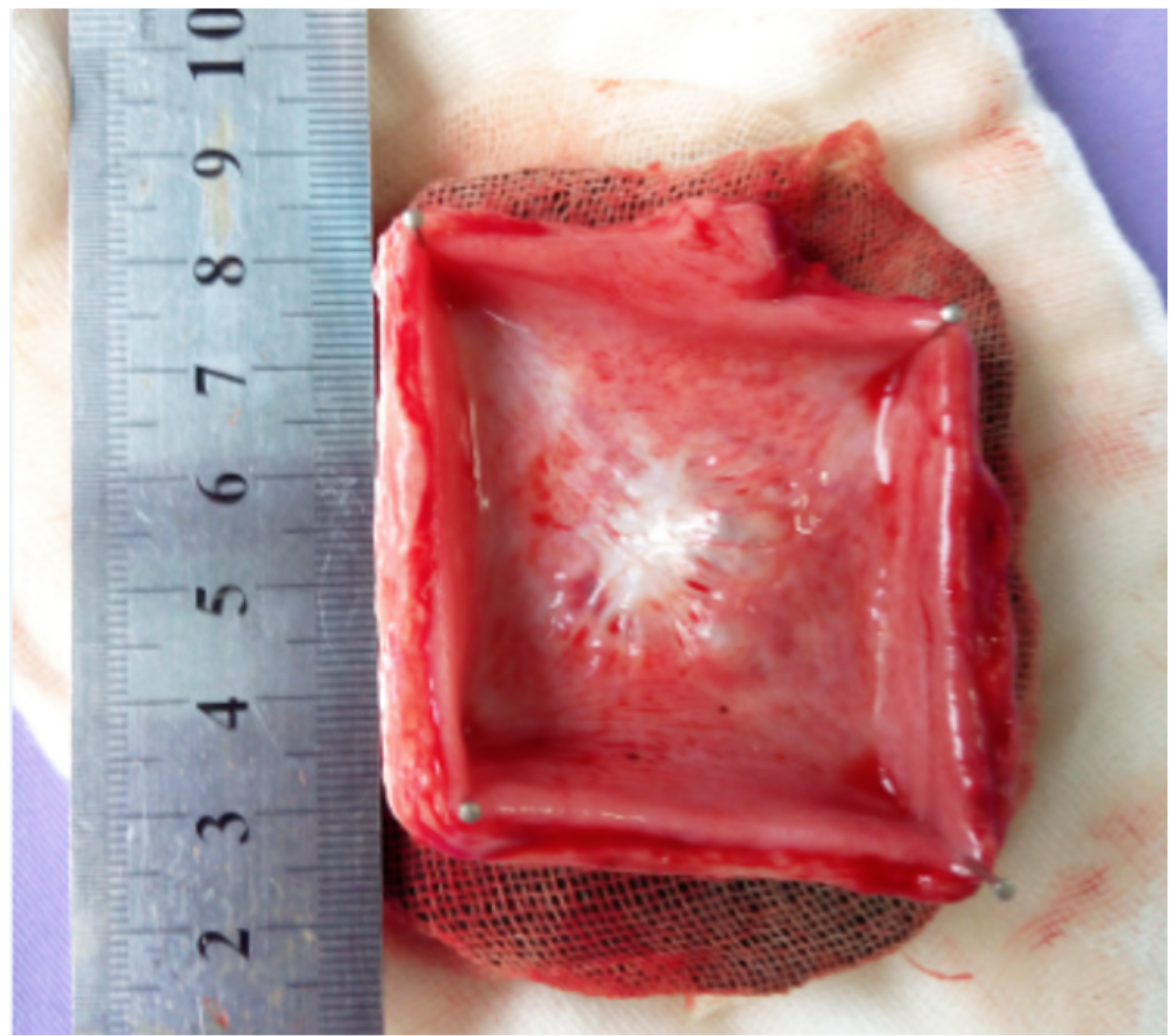

Figure 6

It seems that some augments were contracted in size, but the luminal surface at the resection site appeared soomth and intact. 


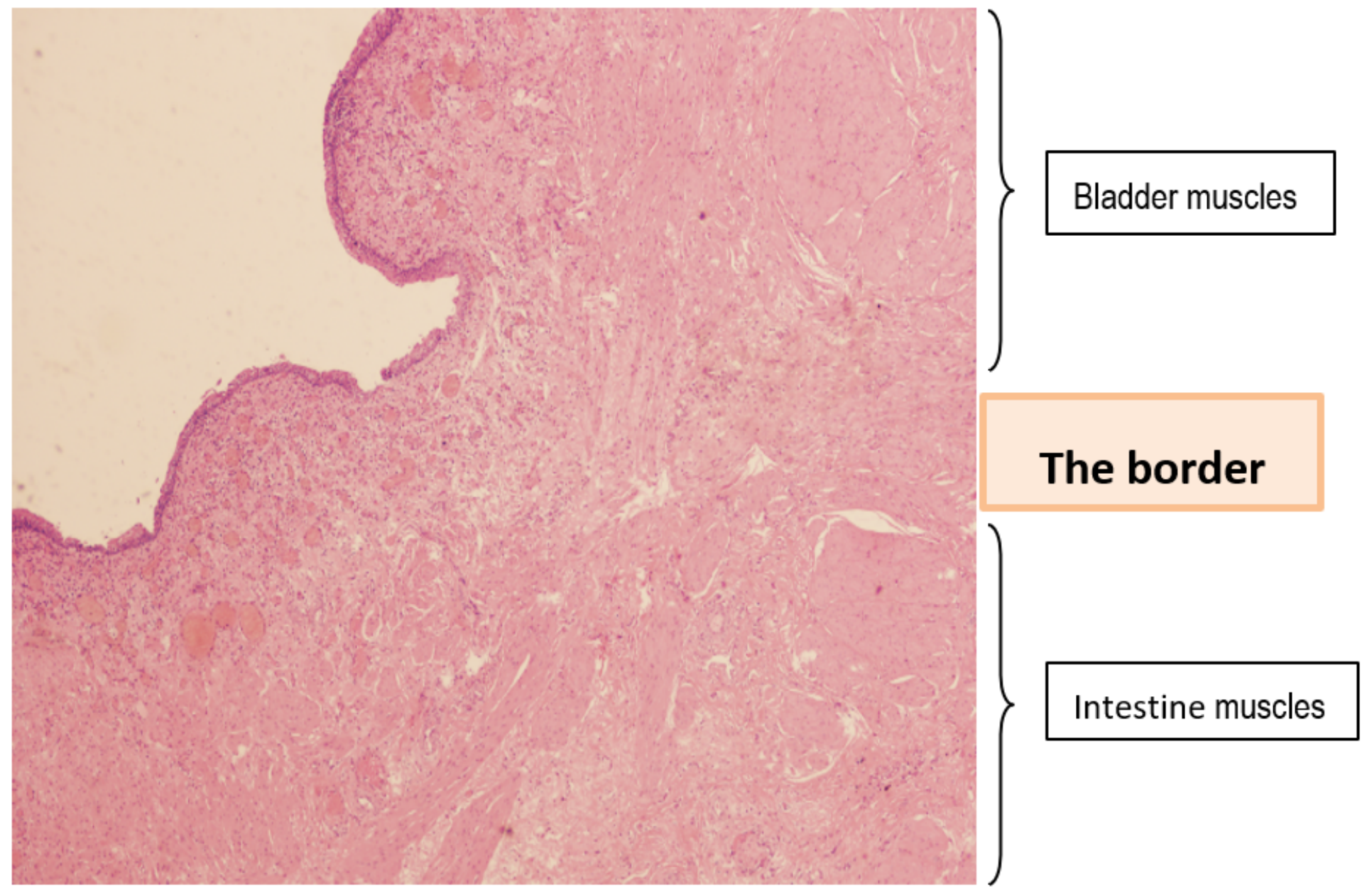

\section{Figure 7}

At week 12 postoperatively, the cavosurface of the bladder reconstructed is covered by transitional cells

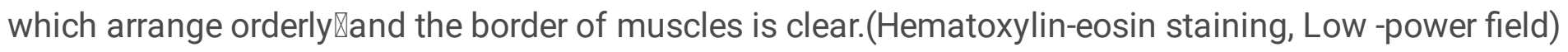




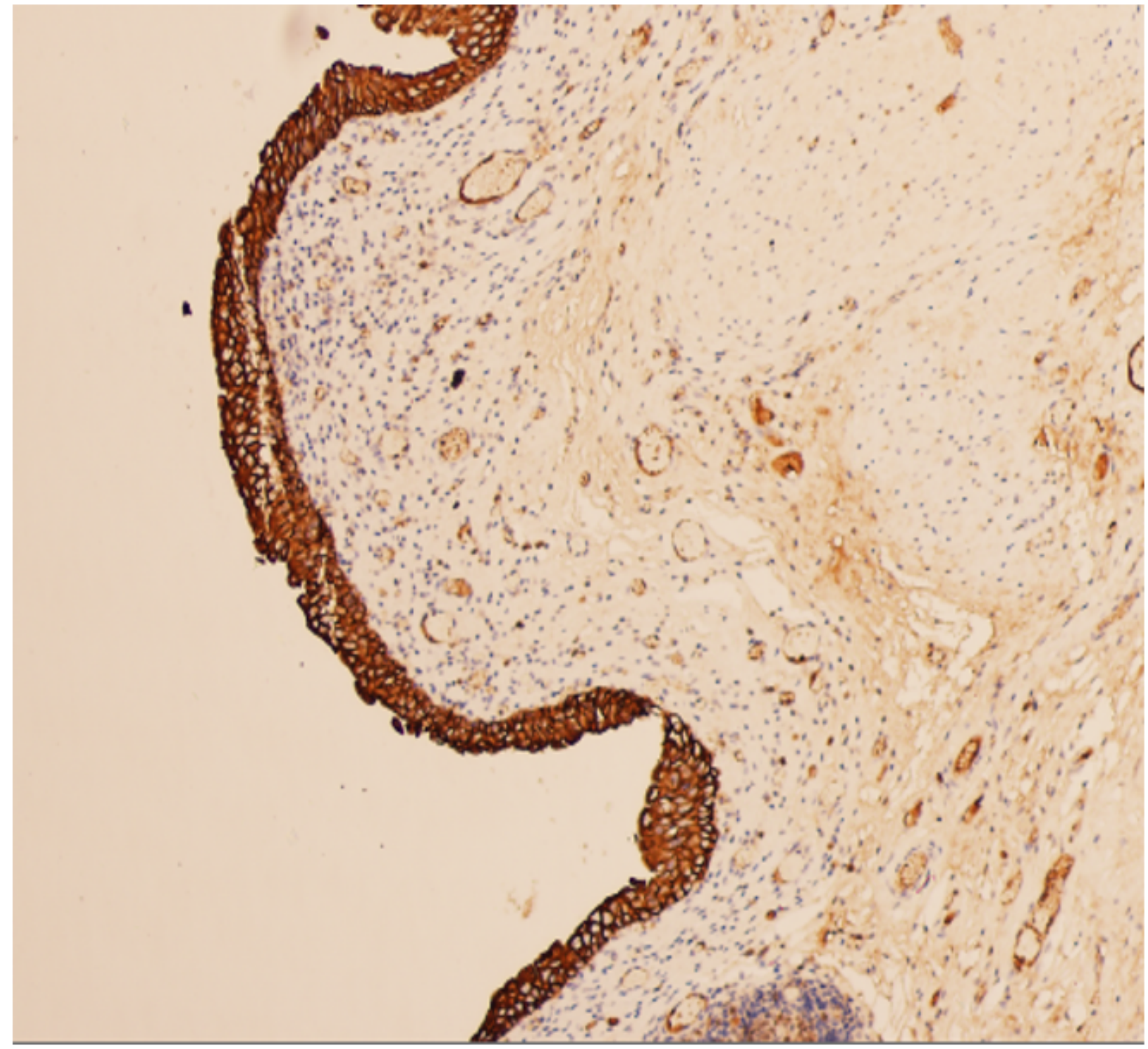

Figure 8

CK7 shows the epithelium brawn stain, and strongly positive all. The epithelium is covered by transitional cell which arrange on 3-5 layers orderly. (Immunohistochemistry staining, Medium-power field) 


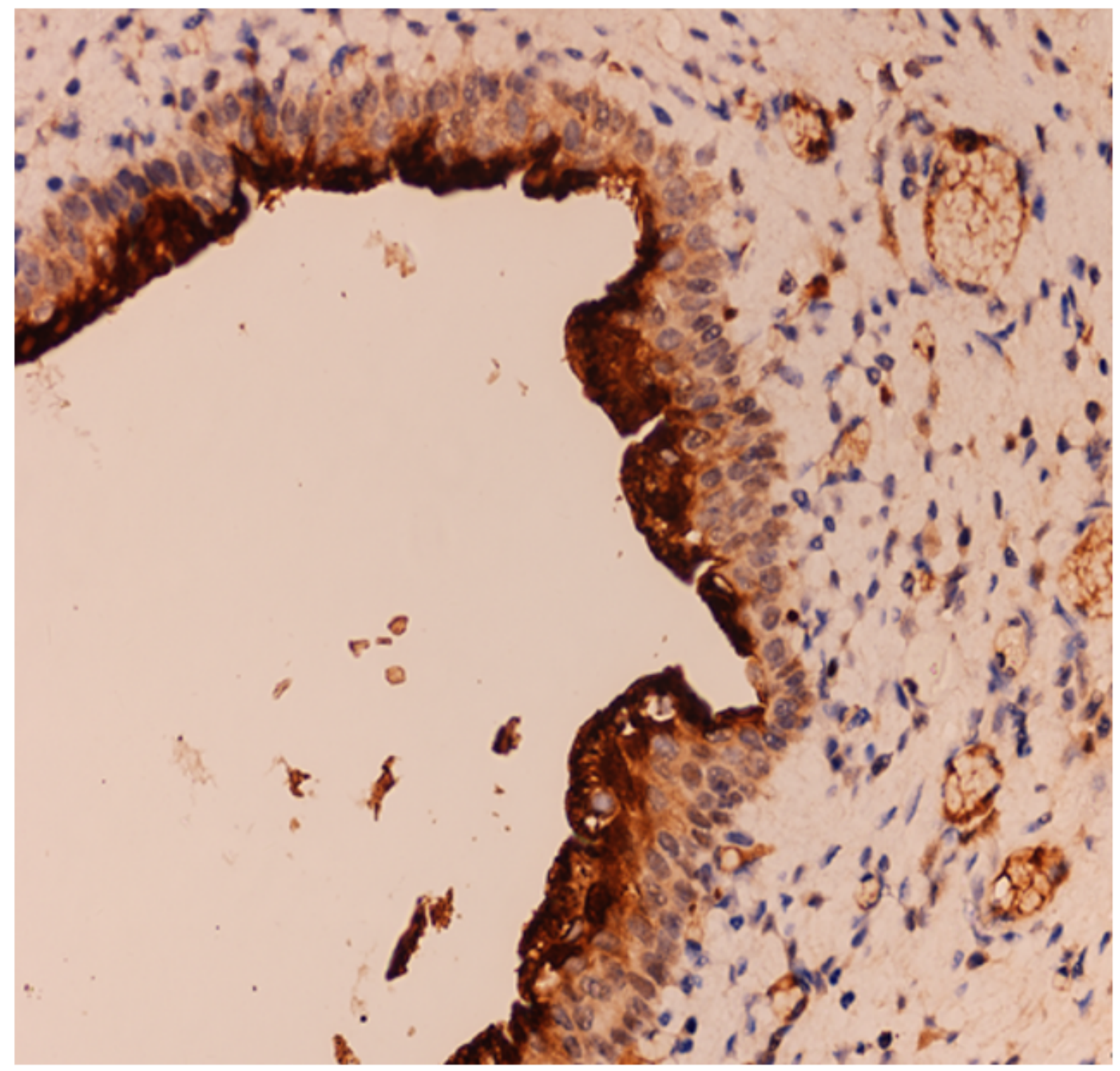

\section{Figure 9}

UPK3a shows the surface cells brawn stain, and strongly positive all. The epithelium arrange on 3-5 layers orderly. (Immunohistochemistry staining, High-power field) 


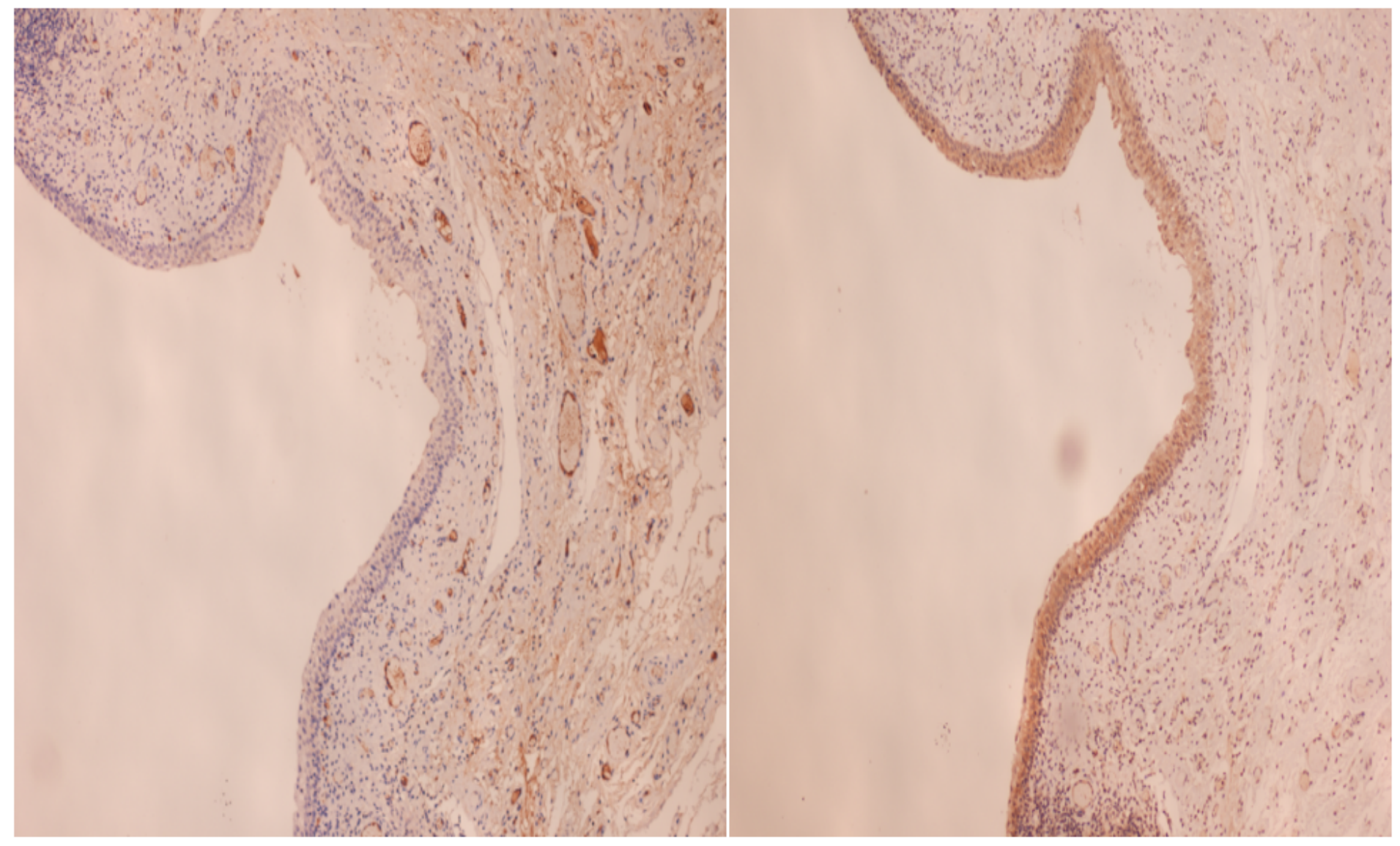

Figure 10

Calretinin(Left) and HBME-1(Right) show the epithelium uncoloured, and negative all, peritoneum transplanted is disappeared. (Immunohistochemistry staining, Medium-power field) 


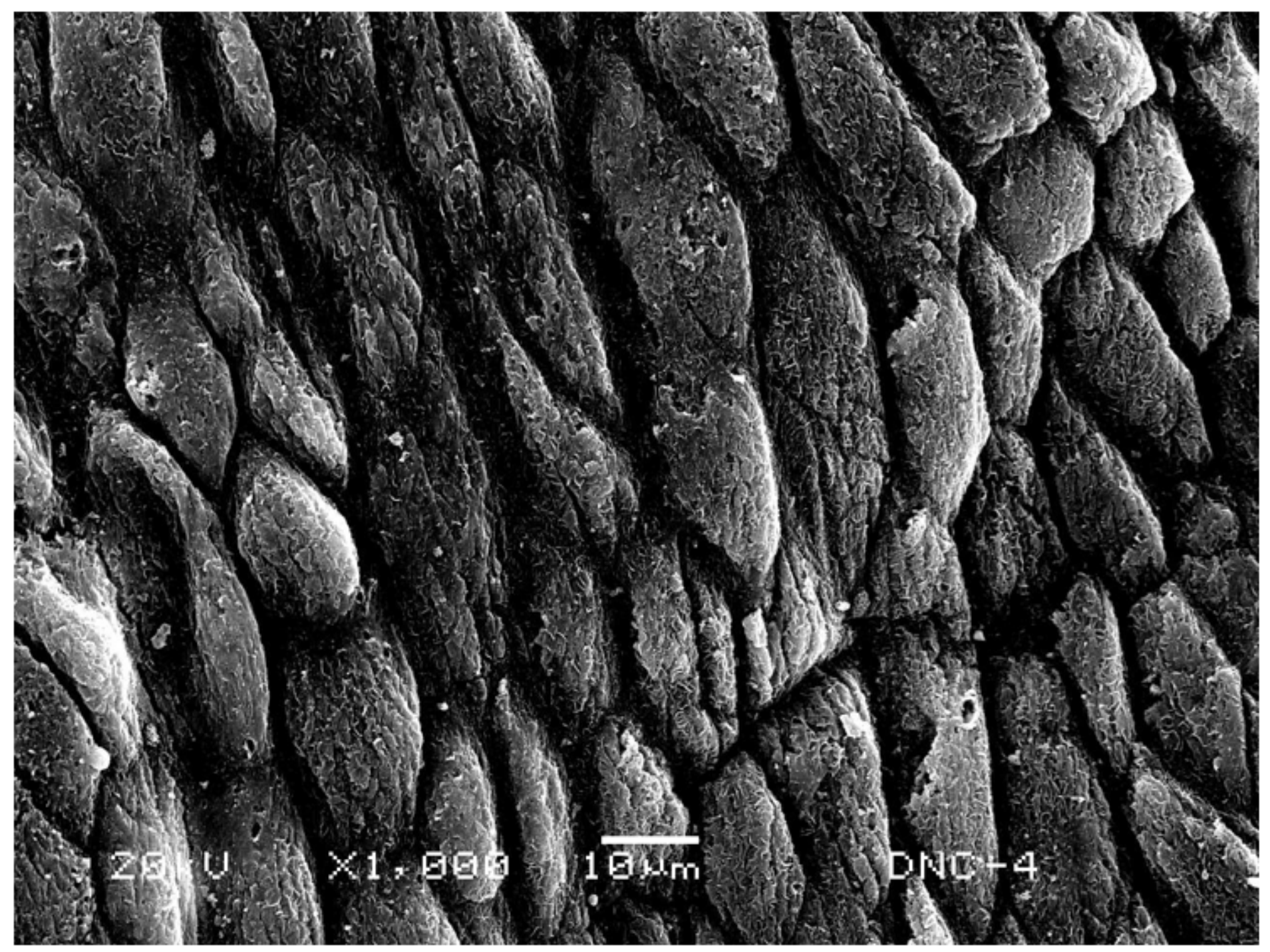

Figure 11

Normal bladder mucosal preoperatively (SEM) 


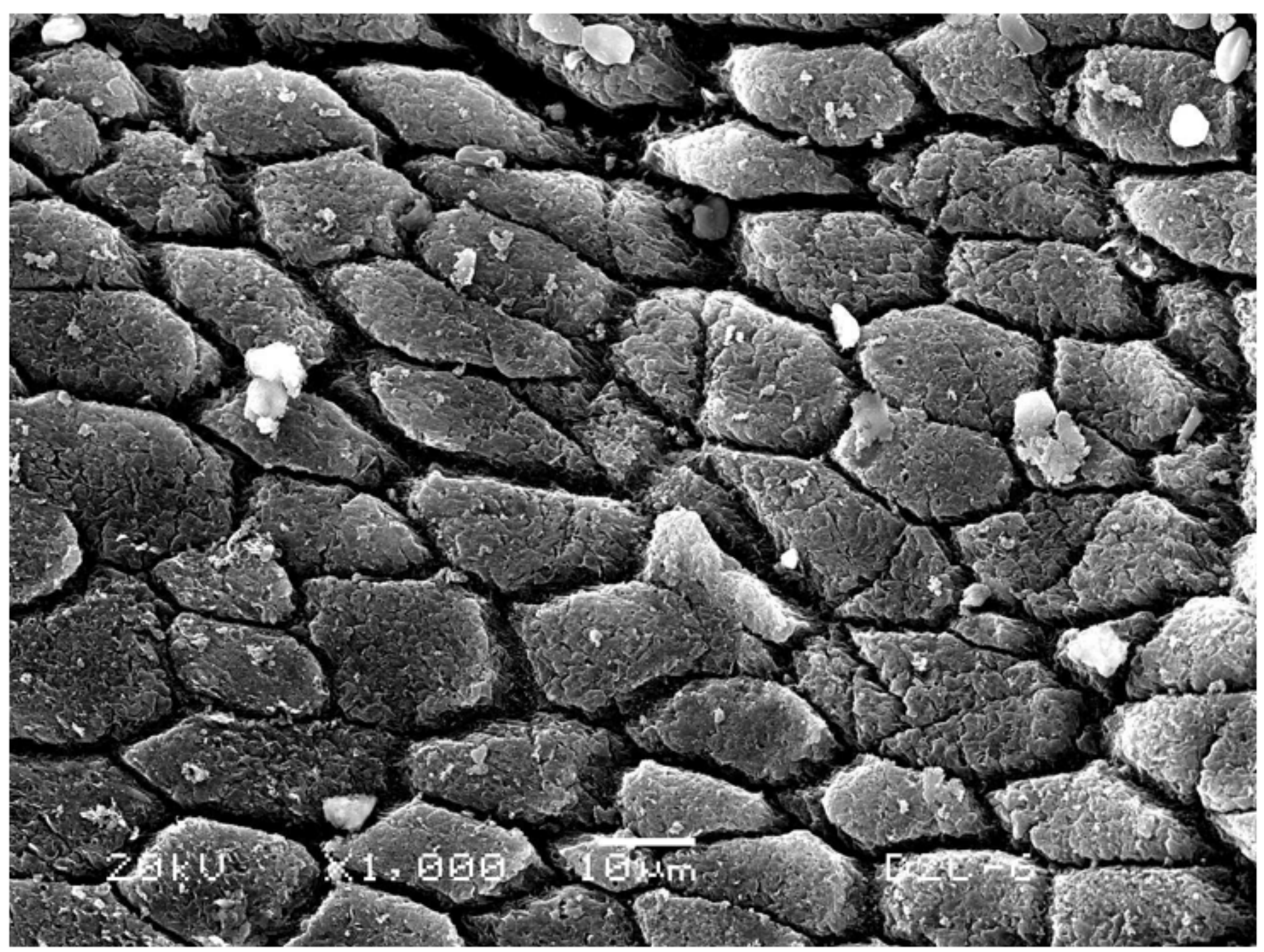

Figure 12

Neobladder mucosal postoperatively 12 weeks (SEM) 


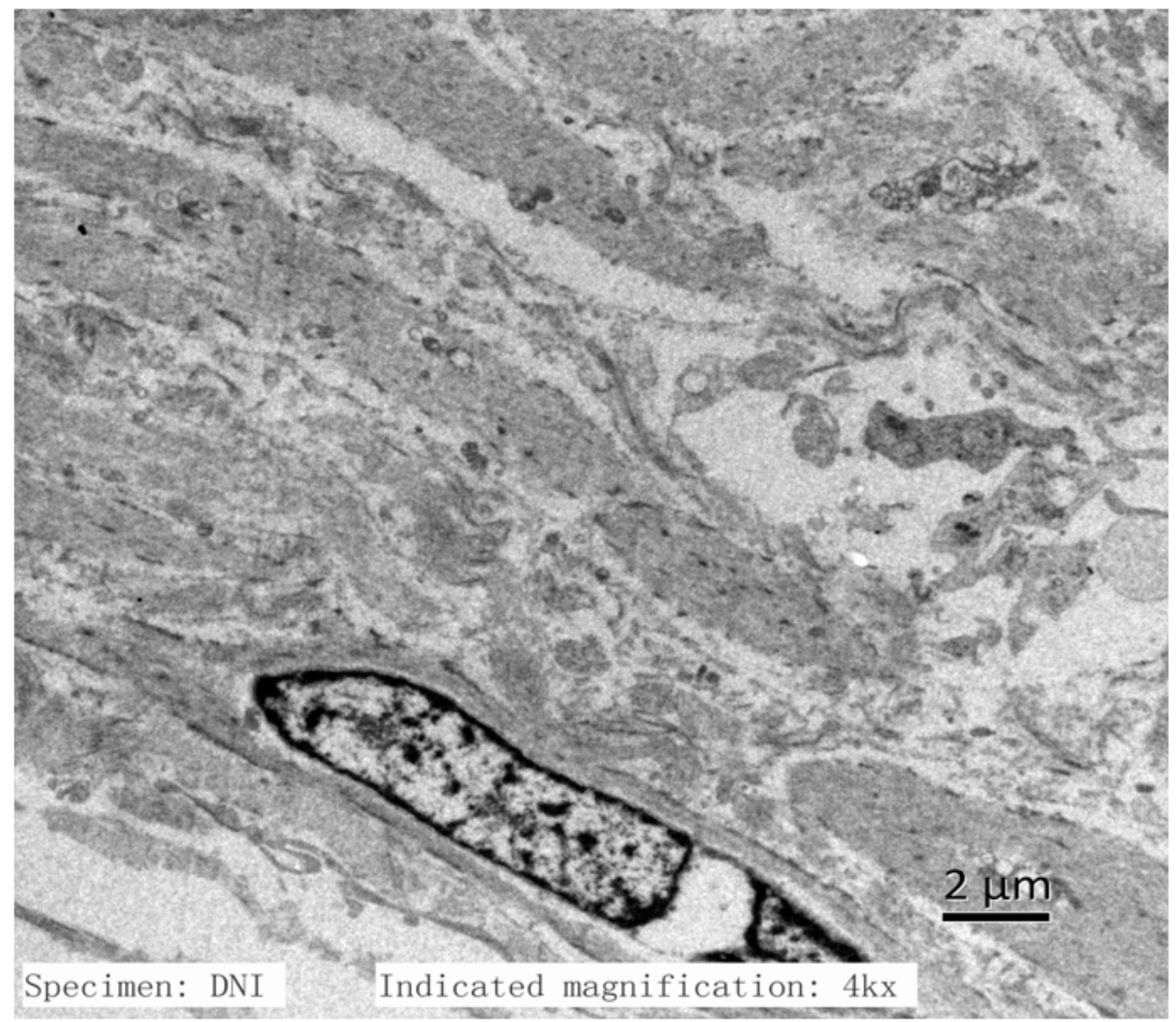

\section{Figure 13}

Normal ileum smooth muscle cells preoperatively (TEM) 


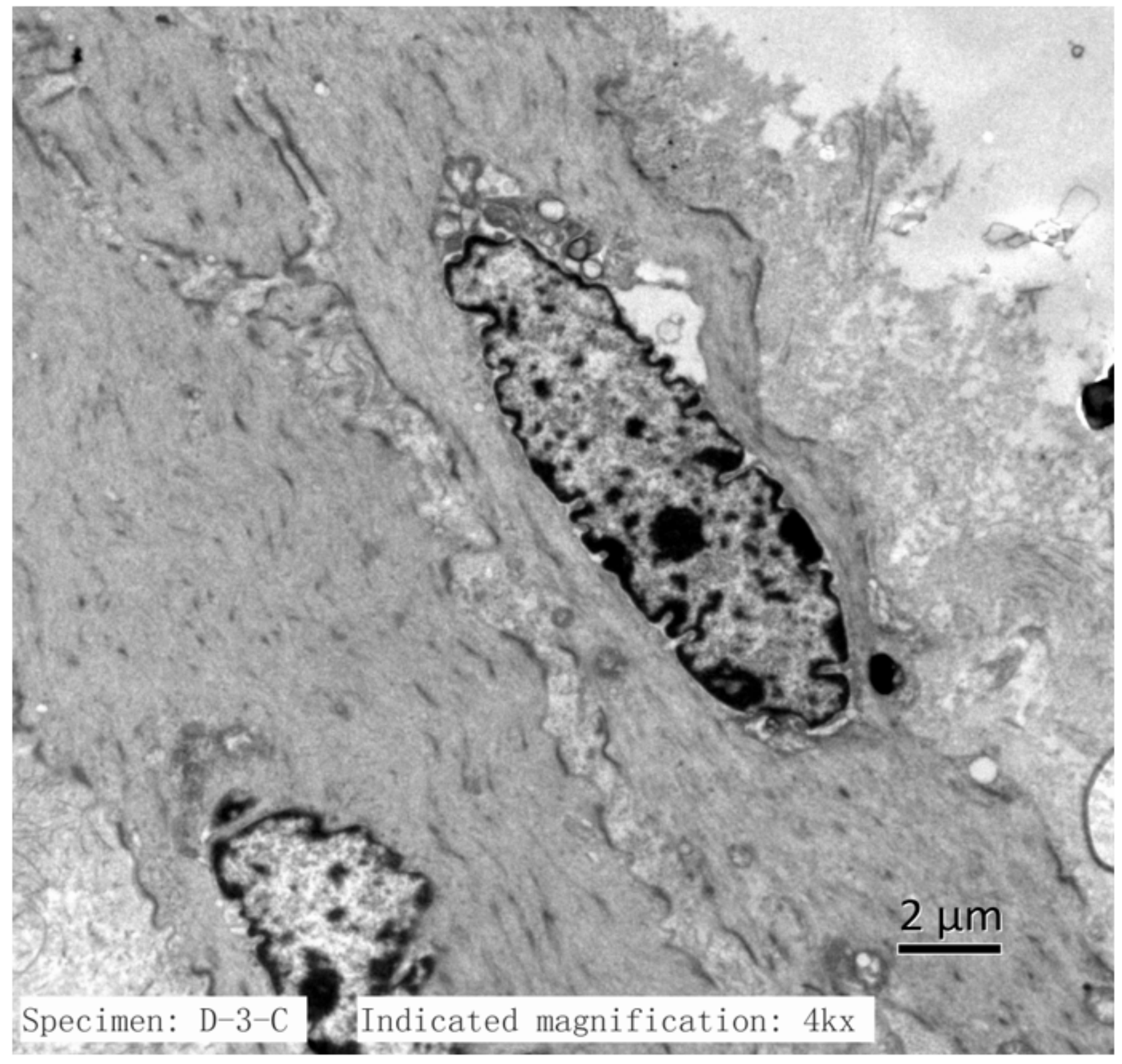

Figure 14

Neobladder smooth muscle cells at postoperatively 12 weeks (TEM) 